\title{
SYMPLECTIC ACTIONS ON COMPACT MANIFOLDS
}

\author{
SOL SCHWARTZMAN
}

(Communicated by Yingfei Yi)

Dedicated to the Memory of Lew Pakula

\begin{abstract}
Let $M^{2 n}$ be a compact connected Kähler manifold. We prove two theorems that in this case imply the following:

(1) If we have a symplectic action of a compact connected Lie group $G$ on $M^{2 n}$ and there exists an orbit under the action of $G$ that is contained in a subset $A$ of $M^{2 n}$ whose first Betti number equals zero, then our action is Hamiltonian.

(2) If we have a continuous symplectic flow on $M^{2 n}$ that preserves some invariant metric, then exactly one of the following statements is true:

(a) Our flow is Hamiltonian.

(b) Our flow has a continuous eigenfunction which is not invariant under the flow.
\end{abstract}

\section{INTRODUCTION}

In what follows $M^{2 n}$ will be a compact connected symplectic manifold whose closed nowhere singular 2-form will be denoted by $w$. We will let $T^{k}$ be the $k$ dimensional torus gotten by taking $\mathbb{R}^{k}$ modulo the subgroup of points with integral coordinates. For any closed 1 -form $\alpha$ on $M^{2 n}$ define $h(\alpha)$ to equal $\alpha \wedge w^{n-1}$. Then $h$ induces a homomorphism $\bar{h}$ from $H^{1}\left(M^{2 n}, \mathbb{R}\right)$ to $H^{n-1}\left(M^{2 n}, \mathbb{R}\right)$. We will say our manifold is of type $L_{1}$ provided $\bar{h}$ is an isomorphism. If $M^{2 n}$ is a Kähler manifold the Hard Lefschetz Theorem tells us that this is the case.

For what we are going to do we will need to know some facts about asymptotic cycles.

If we are given a continuous map $f$ of $[0, \infty)$ into a compact polyhedron $\mathrm{X}$, for each $T>0$ we can pick a curve $C_{T}$ going from $f(T)$ to $f(0)$ so that there is a uniform bound on the lengths of these curves with respect to some metric. Let $A_{T}$ be the curve gotten by tacking on to the curve given by $f$ restricted to $[0, T]$ the $C_{T}$ we have chosen. Let $\bar{A}_{T}$ be the element of $H_{1}(\mathrm{X}, \mathbb{R})$ determined by the closed curve $A_{T}$. If $\lim _{T \rightarrow \infty} \frac{1}{T} \bar{A}_{T}$ exists it can be shown to be independent of the particular curves $C_{T}$ we have chosen. If this limit exists we call it the asymptotic cycle determined by $f$.

Suppose we are given a continuous flow on a compact polyhedron. If $p$ is any quasi-regular point [3] and we take for $f$ the positive semi-orbit emanating from $p$, then there will be an asymptotic cycle which we will denote by $A_{p}$ associated with $f$. If $\mu$ is any invariant probability measure and we let $Q$ be the set of all

Received by the editors November 13, 2012 and, in revised form, March 26, 2013.

2010 Mathematics Subject Classification. Primary 37Jxx, 37J10. 
quasi-regular points we can define the $\mu$-asymptotic cycle $A_{\mu}$ to equal $\int_{Q} A_{p} d \mu(p)$. This makes sense because it will always be true that $\mu(Q)=1$.

In [5] it is shown that if the flow is recurrent then $A_{\mu}$ is the same for all $\mu$. If our flow preserves an invariant metric it is certainly the case that it is recurrent 1 .

When $T^{k}$ acts continuously on $M^{2 n}$ it is well known that there is an invariant metric. Moreover every point $p$ is known to be quasi-regular under the action of any one-parameter subgroup of $T^{k}$. Thus the asymptotic cycle under the action of such a subgroup is defined for each $p$ and is independent of $p$. If for any $v$ in the Lie algebra of $T^{k}$ we let $V$ be the vector field on $M^{2 n}$ we get from the oneparameter subgroup of $T^{k}$ determined by $v$, then for each $p \in M^{2 n}$ the asymptotic cycle $A_{p}^{V}$ is defined and it is the same for all $p$. Hence for any given $v$ and all invariant probability measures $\mu$ for the action of $T^{k}$ on $M^{2 n}$ the asymptotic cycles $A_{\mu}^{V}$ depend only on $V$ and not on $\mu$.

In case the real line $\mathbb{R}$ acts on a compact differentiable manifold $X^{n}$ and we are given an everywhere positive $n$-form $\Omega$ on $X^{n}$ that is invariant under our flow, there will be associated with $\Omega$ an invariant measure $m$. Suppose that $m$ is a probability measure. Then it has been shown by Arnold that the Poincaré dual of the asymptotic cycle $A_{m} \in H_{1}\left(X^{n}, \mathbb{R}\right)$ is the element of $H^{n-1}\left(X^{n}, \mathbb{R}\right)$ corresponding to the $(n-1)$-form $V\lrcorner \Omega$, where $V$ is the vector field defining the flow.

Recall now that our action of $T^{k}$ on $M^{2 n}$ is said to be Hamiltonian if each oneparameter subgroup of $T^{k}$ defines a Hamiltonian flow while the action is symplectic if each such action of a one-parameter subgroup is symplectic.

We are prepared to prove

Lemma 1. Assume $M^{2 n}$ is of type $L_{1}$ and that $V$ is a vector field on $M^{2 n}$ defining a flow for which the measure $m$ associated with $w^{n}$ is an invariant probability measure. Then our flow is Hamiltonian if and only if the asymptotic cycle $A_{m}$ equals zero.

Proof. By the theorem of Arnold cited above, $A_{m}$ equals zero if and only if $\left.V\right\lrcorner w^{n}$ bounds. However $V\lrcorner w^{n}$ equals $\left.n(V\lrcorner w\right) \wedge w^{n-1}$. Since our manifold is of type $L_{1}$, $A_{m}$ equals zero if and only if $\left.V\right\lrcorner w$ bounds. However this exactly says that our flow is Hamiltonian.

Assume now that we are given a symplectic action of $T^{k}$ on $M^{2 n}$.

Theorem 1. If there is a set $A \subseteq M^{2 n}$ with $H^{1}(A, \mathbb{R})=0$ and $A$ contains an entire orbit, then the action of $T^{k}$ is Hamiltonian.

Proof. Since $T^{k}$ preserves a metric the same is true for the flow determined by any 1-parameter subgroup of $T^{k}$. Hence if $V$ is the vector field on $M^{2 n}$ associated with such a flow, the asymptotic cycle $A_{\mu}^{V}$ is the same for all probability measures $\mu$ invariant under the flow.

Let us assume that for some $V_{0}$ arising in this way this common asymptotic cycle is not zero. Since the measure determined by $w^{n}$ is invariant under this flow, the flow is not Hamiltonian.

We are assuming there is an orbit $K$ under the action of $T^{k}$ that is contained in $A$. This orbit is either a single point or a torus, so it is certainly a polyhedron. The flow determined by $V_{0}$ leaves $K$ invariant and there must be at least one invariant probability measure $m$ on $K$ for this flow. Let $\tilde{m}$ be the extension of this measure to $M^{2 n}$ gotten by defining the measure of any set outside $K$ to be zero. 
If $A_{m}^{V_{0}}$ is the asymptotic cycle in $H_{1}(K, \mathbb{R})$ determined by $m$ and $A_{\tilde{m}}^{V_{0}}$ is the asymptotic cycle in $H_{1}\left(M^{2 n}, \mathbb{R}\right)$ determined by $\tilde{m}$, the homomorphism of $H_{1}(K, \mathbb{R})$ into $H_{1}\left(M^{2 n}, \mathbb{R}\right)$ we get from inclusion carries $A_{m}^{V_{0}}$ into $A_{\tilde{m}}^{V_{0}}$. Since our assumption implies that the image of this homomorphism is zero, $A_{\tilde{m}}^{V_{0}}$ must equal zero. Since $A_{\mu}^{V_{0}}$ is independent of $\mu$ we get a contradiction.

In particular, if there is a fixed point for the action of $T^{k}$ we can take as $A$ the set whose only member is this fixed point, so this action is Hamiltonian. This special case is well known.

Suppose now that $M^{2 n}$ is a compact connected differentiable manifold with a non-empty boundary. Without going into detail it is clear that it makes sense to say that we have a closed non-singular 2-form $w$ on $M^{2 n}$ and to assume that $M^{2 n}$ is of type $L_{1}$. Then if we have a symplectic flow on $M^{2 n}$ and there is at least one component of the boundary whose first Betti number equals zero, it will follow that our flow is Hamiltonian, since this component will serve as our set $A$.

We should note that the conclusion of Theorem 1 holds if instead of $T^{k}$ we had assumed our group was any compact connected Lie group. This is because the closure of any 1-parameter subgroup of such a group is a compact connected commutative Lie group and hence is a toral group.

Before proceeding to our next result we need some background material.

Let $C$ be the multiplicative group of complex numbers of absolute value one. If $G$ is a locally compact commutative group, a continuous homomorphism of $G$ into $C$ is called a character of $G$. If such a group $G$ acts continuously on a compact polyhedron $\mathrm{X}$, then a continuous function $F$ from $\mathrm{X}$ into $C$ is called an eigenfunction if there exists a character $\mathcal{X}$ of $G$ such that $F(g \cdot x)=\mathcal{X}(g) F(x)$ for all $x \in \mathrm{X}$ and $g \in G$.

Next let $C(\mathrm{X})$ be the multiplicative group of continuous functions from a polyhedron $\mathrm{X}$ into $C$ and let $R(\mathrm{X})$ be the subgroup of $C(\mathrm{X})$ consisting of those functions in $C(\mathrm{X})$ that have continuous logarithms. It is proved in 9 that if our transformation group $G$ is compact, connected, and commutative, then each equivalence class in the quotient group $C(\mathrm{X}) / R(\mathrm{X})$ contains an eigenfunction.

It is well known that $C(\mathrm{X}) / R(\mathrm{X})$ is naturally isomorphic to $H^{1}(\mathrm{X}, \mathbb{Z})$. Consequently $\operatorname{Hom}(C(\mathrm{X}) / R(\mathrm{X}), \mathbb{R})$ is naturally isomorphic to $\operatorname{Hom}\left(H^{1}(\mathrm{X}, \mathbb{Z}), \mathbb{R}\right)$ and consequently to $H_{1}(\mathrm{X}, \mathbb{R})$.

Suppose now that we have a continuous flow on a compact polyhedron $\mathrm{X}$ that has an invariant metric. Then we will say that an eigenfunction for our flow is non-constant provided it is not invariant under the flow.

Lemma 2. If $p \in \mathrm{X}$ and our flow does not possess any non-constant continuous eigenfunctions, then the asymptotic cycle $A_{p}$ equals zero.

Proof. First recall that because our flow preserves an invariant metric, every point is quasi-regular and $A_{p}$ is the same for all $p \in \mathrm{X}$.

Because our flow preserves an invariant metric the closure in the group of homeomorphisms of X with the $C_{0}$ topology of our 1-parameter group of homeomorphisms is compact in addition to being commutative and connected. If we let $G$ be this closure, we know that every equivalence class in $C(\mathrm{X}) / R(\mathrm{X})$ contains an eigenfunction. The same must therefore be true for our 1-parameter subgroup. Since we are assuming that our flow has no non-constant eigenfunctions, each equivalence class 
in $C(\mathrm{X}) / R(\mathrm{X})$ must contain an invariant function for the action of our 1-parameter group.

It is pointed out in [5] that if $F_{1}$ and $F_{2}$ are two functions in $C(\mathrm{X})$ belonging to the same equivalence class in $C(\mathrm{X}) / R(\mathrm{X})$ and each of them is differentiable with respect to our flow, then for any quasi-regular point $p \in \mathrm{X}$,

$$
\lim _{t \rightarrow \infty} \frac{1}{T} \int_{0}^{T} \frac{F_{1}^{\prime}(t p)}{2 \pi i F_{1}(t p)} d t
$$

equals

$$
\lim _{t \rightarrow \infty} \frac{1}{T} \int_{0}^{T} \frac{F_{2}^{\prime}(t p)}{2 \pi i F_{2}(t p)} d t
$$

Thus for any quasi-regular point $p$ we get a map of $C(\mathrm{X}) / R(\mathrm{X})$ into $\mathbb{R}$. This is seen to be a homomorphism.

Thus for any quasi-regular point $p$ we get an element of $\operatorname{Hom}(C(\mathrm{X}) / R(\mathrm{X}), \mathbb{R})$. We have observed that this group is naturally isomorphic to $H_{1}(\mathrm{X}, \mathbb{R})$. The definition of the asymptotic cycle $A_{p}$ given in [9] was that it is the element of $H_{1}(\mathrm{X}, \mathbb{R})$ we get by our natural isomorphism. This is consistent with the definition given in the introduction of this paper.

Since each equivalence class in $C(\mathrm{X}) / R(\mathrm{X})$ contains an invariant function, our element of $\operatorname{Hom}(C(\mathrm{X}) / R(\mathrm{X}), \mathbb{R})$ determined by $p$ must be zero.

Finally suppose that $M^{2 n}$ is a compact connected symplectic manifold of type $L_{1}$ and that we are given a flow on $M^{2 n}$ that has an invariant metric and is symplectic. Then

Theorem 2. Exactly one of the following statements is true:

(a) Our flow is Hamiltonian.

(b) Our flow has a non-constant eigenfunction.

Proof. Note first that if we know our flow is Hamiltonian, then it has a fixed point and therefore cannot have a non-constant eigenfunction. Thus we need only show that if there is no non-constant eigenfunction the flow is Hamiltonian. However we have just shown that the asymptotic cycle $A_{p}$ equals zero for all $p$ in this case. Then Lemma 1 tells us that our assumptions imply that our flow is Hamiltonian.

Corollary 1. If the assumptions for Theorem 2 hold and $M^{2 n}$ does not fibre over the circle, our flow must be Hamiltonian.

\section{References}

[1] J. de Vries, Elements of topological dynamics, Mathematics and its Applications, vol. 257, Kluwer Academic Publishers Group, Dordrecht, 1993. MR1249063 (94m:54098)

[2] D. McDuff and D. Salamon, Introduction to symplectic topology, Oxford Mathematical Monographs, Oxford Science Publications, The Clarendon Press, Oxford University Press, New York, 1995. MR1373431 (97b:58062)

[3] John C. Oxtoby, Ergodic sets, Bull. Amer. Math. Soc. 58 (1952), 116-136. MR0047262 $(13,850 \mathrm{e})$

[4] Álvaro Pelayo and San Vũ Ngọc, Symplectic theory of completely integrable Hamiltonian systems, Bull. Amer. Math. Soc. (N.S.) 48 (2011), no. 3, 409-455, DOI 10.1090/S0273-0979-201101338-6. MR2801777 (2012i:37097)

[5] Sol Schwartzman, Asymptotic cycles, Ann. of Math. (2) 66 (1957), 270-284. MR.0088720 $(19,568 \mathrm{i})$ 
[6] Sol Schwartzman, Higher dimensional asymptotic cycles, Canad. J. Math. 55 (2003), no. 3, 636-648, DOI 10.4153/CJM-2003-026-0. MR.1980617 (2004d:57036)

[7] Sol Schwartzman, Asymptotic Cycles, Scholarpedia. Available online at http://www . scholarpedia.org/article/Asymptotic_cycles, 2008.

[8] Sol Schwartzman, Asymptotic cycles for actions of Lie groups, Proc. Amer. Math. Soc. 141 (2013), no. 5, 1673-1677, DOI 10.1090/S0002-9939-2012-11445-5. MR3020854

[9] Sol Schwartzman, A split action associated with a compact transformation group, Proc. Amer. Math. Soc. 83 (1981), no. 4, 817-824, DOI 10.2307/2044260. MR630061 (83c:57017)

Department of Mathematics, University of Rhode Island, Kingston, Rhode Island 02881

E-mail address: solschwartzman@gmail.com 